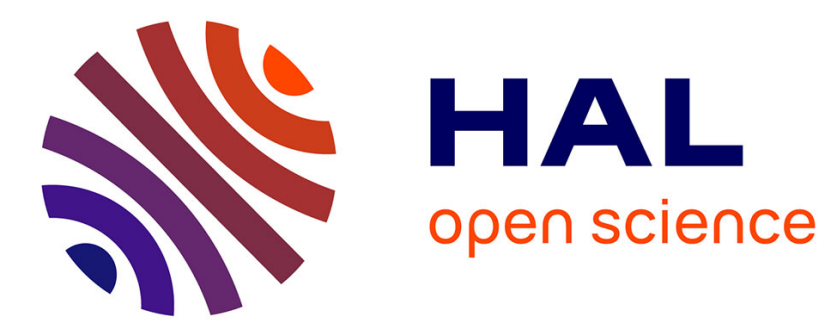

\title{
Design of a haptic guidance solution for assisted power wheelchair navigation
}

Louise Devigne, François Pasteau, Marie Babel, Vishnu K Narayanan, Sylvain Guegan, Philippe Gallien

\section{- To cite this version:}

Louise Devigne, François Pasteau, Marie Babel, Vishnu K Narayanan, Sylvain Guegan, et al.. Design of a haptic guidance solution for assisted power wheelchair navigation. SMC2018 - IEEE International Conference on Systems, Man, and Cybernetics, Oct 2018, Miyazaki, Japan. pp.3231-3236, 10.1109/SMC.2018.00547 . hal-01866150

\section{HAL Id: hal-01866150 \\ https://hal.inria.fr/hal-01866150}

Submitted on 3 Sep 2018

HAL is a multi-disciplinary open access archive for the deposit and dissemination of scientific research documents, whether they are published or not. The documents may come from teaching and research institutions in France or abroad, or from public or private research centers.
L'archive ouverte pluridisciplinaire HAL, est destinée au dépôt et à la diffusion de documents scientifiques de niveau recherche, publiés ou non, émanant des établissements d'enseignement et de recherche français ou étrangers, des laboratoires publics ou privés. 


\title{
Design of a haptic guidance solution for assisted power wheelchair navigation
}

\author{
Louise Devigne ${ }^{1,3}$, François Pasteau ${ }^{3}$, Marie Babel $^{3}$, \\ Vishnu K. Narayanan ${ }^{2}$, Sylvain Guégan ${ }^{4}$ and Philippe Gallien ${ }^{1}$
}

\begin{abstract}
Smart powered wheelchairs can increase mobility and independence for people with disability by providing navigation support. This support can be supplied in the form of autonomous or semi-autonomous obstacle avoidance systems. However, for rehabilitation or learning purposes, it would be of great benefit for wheelchair users to have a better understanding of the surrounding environment while driving. Therefore, another way of providing navigation support is to communicate information through a dedicated and adapted feedback interface. We here propose a framework in which feedback is provided by sending forces through the wheelchair controller as the user steers the wheelchair. This solution is based on a low complex optimization framework able to perform smooth trajectory correction and to provide obstacle avoidance. The impact of the proposed haptic guidance solution on user driving performance was assessed during this pilot study for validation purposes through an experiment with 4 able-bodied participants. They were asked to drive a power wheelchair on an obstacle course with and without activation of the force feedback. Results of this pilot study showed that the number of collisions significantly decreased while force feedback was activated, thus validating the proposed framework.
\end{abstract}

\section{INTRODUCTION}

The use of a power wheelchair for individuals with mobility impairments has positive outcomes such as enhancing user autonomy and social inclusion [1][2]. However, heavy motor and/or perceptual impairments can lead to a variety of activity limitations [3]. In particular, perceptual impairments can impact the ability to independently operate a wheelchair safely [4]. In this context, several research teams developed autonomous or semi-autonomous wheelchair navigation solutions [5][6].

Although such systems assist the user while driving a wheelchair, providing feedback information (visual, auditory or haptic/tactile) could have additional benefits for learning and rehabilitation purposes [7].

In the robotics field, feedback information is often communicated through haptic interfaces, especially for human robot interaction applications. Indeed, applying forces, vibrations, or motions to the user interface allows to communicate information between a user and a remote environment. Typically, this can be useful for robot teleoperation, interaction with a virtual environment or even remote surgery [8][9][10]. This

\footnotetext{
${ }^{1}$ Rehabilitation Center Pôle Saint Hélier, Rennes, France.

2 ATR Intelligent Robotics and Communication Labs, Kyoto, Japan.

${ }^{3}$ Univ Rennes, INSA, CNRS, Inria, Irisa-UMR6074, F-35000 Rennes, France.

${ }^{4}$ Univ Rennes, INSA, CNRS, Inria, LGCGM-EA3913, F-35000 Rennes, France.
}

type of feedback is typically used for people with visual impairments [11][12], where haptic information compensates for the lack of visual information and thus allows the user to improve his/her interaction with the external environment. Some studies have also shown the benefits of using haptic feedback for motor rehabilitation process [13][14].

It is then possible to create a communication channel between the user and the system he/she is using. This feedback provides additional information to users during their interaction such that their experience or their performance can be enhanced. Moreover, as such a feedback can improve user understanding while performing a given task, haptic feedback could be seen as an effective rehabilitation tool to train severely disabled people to drive a powered wheelchair [15].

Research works have been done on the use of haptic feedback for power wheelchair driving but are only focused on a specific line following task [16][17]. Moreover, several teams investigated force feedback for power wheelchair driving. In [18], the authors evaluated the impact of force feedback on user performance while driving a power wheelchair in a virtual environment. Results of trials with people with disability showed fewer collisions while the feedback was activated. Vander Poorten et al. [19] also developed a haptic feedback algorithm for power wheelchair driving relying on the definition of a collision-free paths computed from a local map of the environment. Such systems typically use Potential Field Methods relying on the definition of a local map of the environment requiring information classically measured by expensive sensors such as laser range finder.

In this paper we present a novel approach for power wheelchair haptic navigation assistance which uses low-cost distance sensors and does not require to compute a local map of the environment. This solution relies on a sensor-based servoing approach we presented in [20]. In this framework, the force feedback information is computed from the fusion of user manual control and sensor-based constraints without any a priori knowledge of the environment. A reactive force feedback is thus provided to the user while driving.

This paper is organized as follows: section II describes the force feedback algorithm. Section III presents a simulation of the system in a virtual environment. Section IV determines first the experimental setup. Then we present the pilot study experiment we performed with able-bodied participants. Section VII presents the results of the experiment. 


\section{Modeling}

The wheelchair is modeled as a 6 wheeled robot composed of 2 actuated wheels and 4 passive caster wheels. It is controlled with 2 variables, namely the linear velocity $u$ and the rotational velocity $\omega$. As shown on Fig. 1, we define the following notations

- let $\mathbf{u}=\left[\begin{array}{ll}u & \omega\end{array}\right]^{T}$ be the velocity of the wheelchair with $u$ and $\omega$ respectively the linear and angular velocities,

- let $\mathbf{v}_{s_{i}}=\left[\begin{array}{ll}u_{s_{i}} & \omega_{s_{i}}\end{array}\right]^{T}$ be the velocity of the sensor $s_{i}$ with $u_{s_{i}}$ and $\omega_{s_{i}}$ respectively the linear and angular velocities of the sensor $s_{i}$,

- let $x_{i}$ be the distance from the sensor $s_{i}$ to the obstacle,

- let $\theta_{i}$ be the orientation of the sensor $s_{i}$ with respect to the robot frame $\mathrm{z}$-axis,

- let $e_{i}$ be the difference between the measured distance $x_{i}$ and its desired value $x_{i}^{*}$.
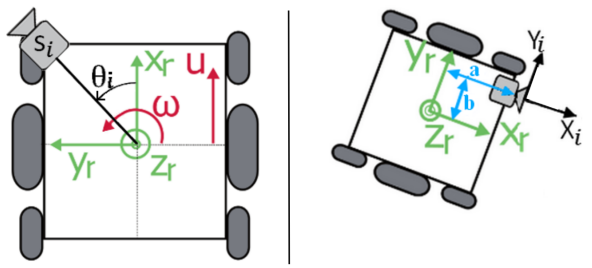

Fig. 1: Definition of the robot frame.

The translation between the sensor $s_{i}$ frame and the robot frame is given by ${ }^{r} \mathbf{t}_{s_{i}}=\left[\begin{array}{lll}a & b & 0\end{array}\right]^{T}$ with $a$ and $b$ depicted on Fig. 1. The rotation matrix relative to the sensor $s_{i}$ frame with respect to the robot frame ${ }^{s_{i}} \mathbf{R}_{r}$ is given by

$$
{ }^{s} \mathbf{R}_{r}=\left[\begin{array}{ccc}
\cos \theta_{i} & \sin \theta_{i} & 0 \\
-\sin \theta_{i} & \cos \theta_{i} & 0 \\
0 & 0 & 0
\end{array}\right] .
$$

From ${ }^{s_{i}} \mathbf{R}_{r}$ and ${ }^{s_{i}} \mathbf{t}_{r}$, the velocity screw transformation matrix is given by

$$
{ }^{s_{i}} \mathbf{W}_{r}=\left[\begin{array}{cc}
{ }^{s_{i}} \mathbf{R}_{r} & {\left[{ }^{s_{i}} \mathbf{t}_{r}\right]_{\times}{ }^{s_{i}} \mathbf{R}_{r}} \\
\mathbf{0}_{3 \times 3} & { }^{s_{i}} \mathbf{R}_{r}
\end{array}\right] .
$$

with []$_{\times}$the skew matrix expression. The velocity of the sensor $s_{i}$ in the sensor frame $\mathbf{v}_{s_{i}}$ is derived from the robot velocity $\mathbf{u}$ in the robot frame using

$$
\mathbf{v}_{s_{i}}={ }^{s_{i}} \mathbf{T}_{r} \mathbf{u}
$$

with

$$
{ }^{s_{i}} \mathbf{T}_{r}={ }^{s_{i}} \mathbf{W}_{r}{ }^{r} \mathbf{J}_{r},
$$

where ${ }^{r} \mathbf{J}_{r}$ is the robot jacobian expressed in the robot frame:

$$
{ }^{r} \mathbf{J}_{r}=\left[\begin{array}{llllll}
1 & 0 & 0 & 0 & 0 & 0 \\
0 & 0 & 0 & 0 & 0 & 1
\end{array}\right]^{T} .
$$

\section{METHOD}

In order to assist a user in controlling a wheelchair, we can communicate additional information by using haptic control devices which provide feedback forces to the user's hand. This way, the user benefits from a communication channel with the wheelchair controller.

\section{A. Assistive algorithm computation}

We can define an interaction matrix $\mathbf{L}_{x_{i}}$ for each sensor $s_{i}$ such that

$$
\dot{x}_{i}=\mathbf{L}_{x_{i}} \mathbf{v}_{s_{i}} .
$$

In order to modify a trajectory leading to a collision, we design a proportional corrector by constraining $\dot{x}_{i}$ by a minimum value ${ }_{\lambda} e_{s_{i}}$. We then get:

$$
\dot{x}_{i} \geq-\lambda e_{s_{i}} .
$$

The combination of (6) and (7) results in

$$
\mathbf{L}_{x_{i}}{ }^{s_{i}} \mathbf{T}_{r} \mathbf{u} \geq-\lambda e_{s_{i}}
$$

In our case with $\mathrm{N}$ sensors, inequality (8) can be rewritten as

$$
\mathbf{A} \mathbf{u} \geq \mathbf{B}
$$

with $\mathbf{A}=\left[\begin{array}{c}\mathbf{A}_{0} \\ \vdots \\ \mathbf{A}_{N-1}\end{array}\right]$ and $\mathbf{B}=\left[\begin{array}{c}B_{0} \\ \vdots \\ B_{N-1}\end{array}\right]$.

For each sensor $s_{i}$ the following inequation

$$
\mathbf{A}_{i} \mathbf{u} \geq B_{i}
$$

defines a half-plane in the wheelchair velocity domain. All the computed intersected half-planes define a convex area in the wheelchair velocity domain as shown on Fig. 3. This area is used in the haptic guidance framework which is presented below.

\section{B. Haptic guidance and haptic feedback assistance}

Two types of haptic navigation assistance for power wheelchair driving can be distinguished:

- haptic guidance: consists in assisting the user to safely control the wheelchair. To do so, a forces are communicated to the user through the wheelchair controller to provide additional information about the environment. In this case, the user has full control of the wheelchair and collisions can occur.

- haptic feedback: the force feedback controller is used in conjunction with the obstacle avoidance framework. It aims at improving user understanding of the behavior of the system while correcting the trajectory.

In this paper, we focus on the haptic guidance solution.

\section{Haptic guidance algorithm}

An appropriate information to be delivered to the user while driving would be the direction to which he/she should put the joystick in order to avoid collisions with obstacles. This information can be communicated to the user by sending appropriate forces through the wheelchair controller.

Let $\mathbf{u}_{o p}=\left(u_{o p}, \omega_{o p}\right)$ be the user desired velocity, and $\mathbf{u}_{c m d}=\left(u_{c m d}, \omega_{c m d}\right)$ be the computed robot velocity. The novelty of the proposed haptic guidance framework is to directly compute the force magnitude and direction from 
the wheelchair velocity domain. Let $\overrightarrow{\mathbf{k}}$ be the vector from $\mathbf{u}_{o p}$ to $\mathbf{u}_{c m d}$ in the wheelchair velocity domain (Fig. 3 ). The force feedback vector $\overrightarrow{\mathbf{F}}$ to be applied to the user interface will be computed from this information.

The computation of $\mathbf{u}_{c m d}$ from $\mathbf{u}_{o p}$ under constraint (9) is an optimization problem using

$$
\left\{\begin{aligned}
\mathbf{u}_{c m d} & =\min _{\mathbf{u}} f\left(\mathbf{u}_{o p}, \mathbf{u}\right) \\
\mathbf{A} \mathbf{u} & \geq \mathbf{B}
\end{aligned}\right.
$$

The function $f$ is defined such as

$$
f\left(\mathbf{u}_{o p}, \mathbf{u}\right)=\alpha\left(u-u_{o p}\right)^{2}+\beta\left(\omega-\omega_{o p}\right)^{2}
$$

with $\alpha \geq 0$ and $\beta \geq 0$.

As shown on Fig. 3, we define 2 sets $\mathbf{P}_{0}$ and $\mathbf{P}_{1}$ such as

$$
\begin{aligned}
& \mathbf{P}_{0}=\{\mathbf{u} \mid \mathbf{A u} \geq \mathbf{B}\} \\
& \mathbf{P}_{1}=\{\mathbf{u} \mid \mathbf{A u}>\mathbf{B}\} .
\end{aligned}
$$

Hence a solution of the optimization problem (11) exists only if $\mathbf{P}_{0} \neq \emptyset$. Else, $x_{i}^{*}$ is dynamically decreased to expand the size of $\mathbf{P}_{0}$.

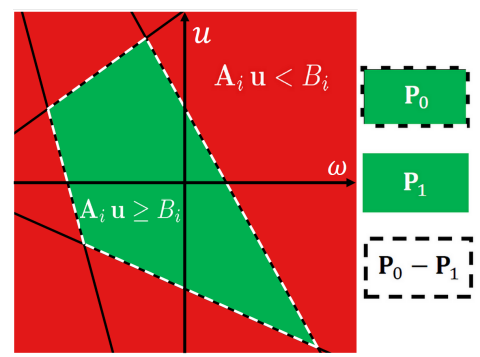

Fig. 2: Definition of $\mathbf{P}_{0}$ and $\mathbf{P}_{1}$. Forbidden and allowed areas are represented respectively in red and green in the wheelchair velocity domain.

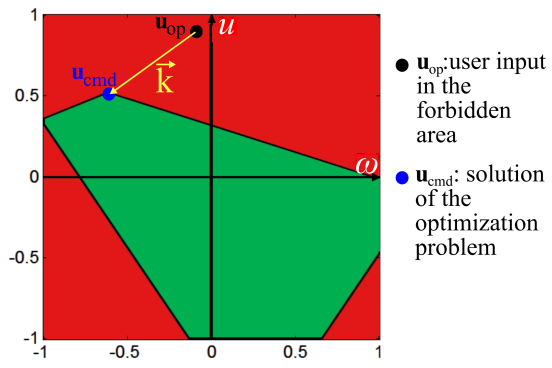

Fig. 3: The algorithm output corresponds to the projection of user input within the allowed area. $\overrightarrow{\mathbf{k}}$ represents the vector from the user input to the algorithm output.

There are two different cases:

1) if $\mathbf{u}_{o p} \in \mathbf{P}_{0}$, then $\mathbf{u}_{c m d}=\mathbf{u}_{o p}$,

2) otherwise, as the cost function is convex and its minimum is reached when $\mathbf{u}=\mathbf{u}_{o p}, \mathbf{u}_{c m d} \in \mathbf{P}_{0}-\mathbf{P}_{1}$. This means that it exists one $i$ for which $\mathbf{A}_{i} \mathbf{u}=B_{i}$.
Here the result $\mathbf{u}_{c m d}$ of this optimization problem is shown in Fig. 3. It results from a search along $\mathbf{P}_{0}-\mathbf{P}_{1}$.

We define $\overrightarrow{\mathbf{k}}\left(r_{k}, \theta_{k}\right)$ and $\overrightarrow{\mathbf{F}}(r, \theta)$ in polar coordinates with respectively $r_{k}$ and $r$ their magnitudes and $\theta_{k}$ and $\theta$ their directions. This representation leads to the following properties:

- force direction is the same as the direction of the vector $\overrightarrow{\mathbf{k}}$ in the wheelchair velocity domain, leading to $\theta=\theta_{k}$, - we define the force magnitude such that $r=\alpha r_{k}$ with

$-\alpha>0$ a proportional gain which can be modified to adapt the force magnitude to the user strength,

- $r_{k}=\|\overrightarrow{\mathbf{k}}\|$ the euclidean distance between $\mathbf{u}_{o p}$ and $\mathbf{u}_{c m d}$ in the wheelchair velocity domain so that

$$
\|\overrightarrow{\mathbf{k}}\|=\sqrt{\left(u_{o p}-u_{c m d}\right)^{2}+\left(\omega_{o p}-\omega_{c m d}\right)^{2}}
$$

This way, the force guides the user towards a joystick position for which the resulting user input is safe. Force magnitude is proportional to the distance between user input and its projection onto the allowed area.

To sum up, a set of constraints deduced from distance sensors is used to defined two areas in the wheelchair velocity domain. Then, if the user input is unsafe (i.e. within the forbidden area), a corresponding risk-free input is calculated by means of a projection within the allowed area. From this, we deduce the force to be sent to the user through the joystick. This force is directed from user input towards the corresponding risk-free input. Its magnitude is proportional to the euclidean distance between user input and the related projection within the allowed area. This way, the user is at the same time notified that there is a danger of collision and guided to a joystick position corresponding to safe input. It is to be emphasized that the user has full control of the wheelchair and can maintain his/her desired joystick position. The information communicated in the form of force feedback is only an indication of the risk-free joystick position the most compliant with his/her intention. Such a solution is then mainly meant to be used for learning or rehabilitation purposes.

\section{Simulations}

In this section, we present the results of the very first step in the development of our haptic guidance solution. It consists in a simulation performed on a simulator for assisted power wheelchair driving we previously developed. This simulator is a flexible tool for development and testing of navigation assistance methods without having to equip a power wheelchair. In this framework, the user operates a force feedback jostick to drive a virtual robotized wheelchair in a virtual environment. The framework has been designed to be directly compliant with a monitor screen display, a Virtual Reality room or a Head Mounted Device and is presented in more details in [21]. 


\section{A. Simulation conditions}

The virtual environment is displayed on a computer screen as it is only used to demonstrate the properties of the proposed haptic guidance solution. The virtual environment used for this simulation is shown on Fig. 4. It consists of a $1.5 \mathrm{~m}$ wide and $10 \mathrm{~m}$ long corridor with 4 obstacles. The virtual wheelchair starts from the beginning of the corridor facing the top wall with an angle of $60^{\circ}$. The wheelchair is here equipped with 22 virtual sensors positioned all around the virtual wheelchair according to Fig. 5b.

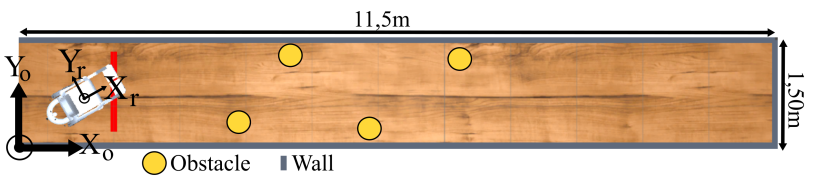

Fig. 4: Top view of the map used for simulation.

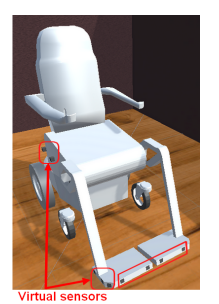

(a) Virtual wheelchair.

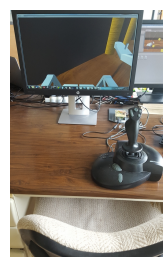

(c) Simulation setup.

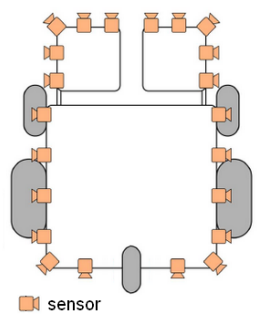

(b) Sensor positioning.

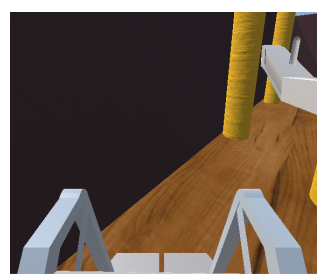

(d) User view during the simulation.
Fig. 5: Virtual environment and simulation setup.

The user is sitting in front of the computer screen and controls the virtual wheelchair (Fig. 5a) by operating a force feedback joystick (Fig. 5c). User view during the simulation is showed in Fig. 5d. The force feedback game controller is a commercially available joystick from Microsoft which we adapted to match wheelchair driving constraints by implementing automatic centering and reducing the number of degrees of freedom to 2 .

In order to emphasize the properties of the proposed force feedback solution, the user voluntary tries to collide with the virtual obstacles while steering the wheelchair.

\section{B. Simulation results}

Simulation results are presented in Fig. 6. Fig. 6a shows the trajectory of the wheelchair during the simulation. Blue arrays represent the force vectors which are sent to the user through the force feedback joystick while driving. Fig.
$6 \mathrm{~b}$ represents the rotational and linear velocity inputs. No collisions were recorded during this simulation.

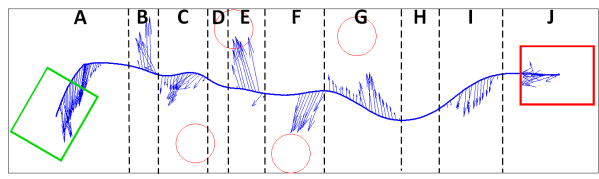

(a) Trajectory of the wheelchair in the virtual environment

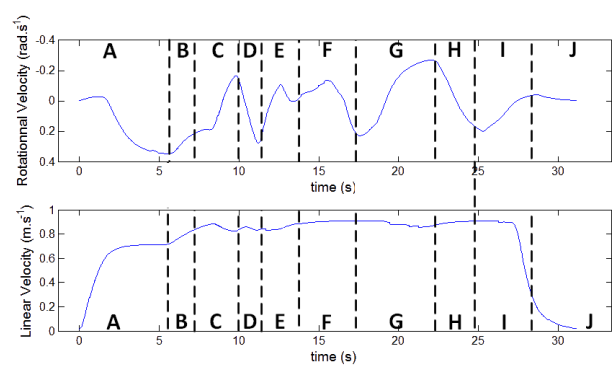

(b) Rotational and linear velocities applied to the wheelchair

Fig. 6: Simulation results.

In Fig. 6, in part $\mathrm{A}$, we observe that as long as the user is driving towards the wall on his left, he is being notified that there is a risk of collision by means of forces directly towards the opposite direction. Then, when the user modifies the angular velocity input, both the magnitude and direction of the force progressively decrease. When the wheelchair becomes parallel to the walls, as there is no risk of collision anymore (typically in $\mathrm{D}$ and $\mathrm{H}$ parts), the user does not receive any force feedback anymore. In $\mathrm{B}$ and $\mathrm{E}$ parts, the user drives towards the obstacle on his right, the algorithm then sends forces towards the left direction. We can observe the impact of this additional information on the angular velocity. Indeed the decreasing of the angular velocity input associated with a slight increase of the linear input shows that the user is adapting his joystick input while receiving force feedback information. We can observe similar results in $\mathrm{C}$ and $\mathrm{F}$ parts where the user drives toward the obstacle on his left.

\section{EXPERIMENT}

As power wheelchair users can have weak upper limbs and/or tremors, it is mandatory to ask able-bodied participants to evaluate our haptic guidance solution before being able to obtain ethical approval regarding trials with people with disabilities. In this pilot study including 4 able-bodied participants, the impact of navigation assistance in the form of haptic guidance solution is assessed.

\section{A. Experimental setup}

We used the same wheelchair as in [22], a power wheelchair which we equipped with the same force feedback joystick as the one used for the simulation. Although it is the same wheelchair, sensor configuration has been upgraded: we empirically determined an optimal configuration composed of 22 sensors. Those sensors were installed on the wheelchair 


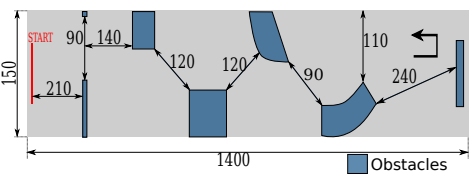

Fig. 7: Obstacle course, values correspond to distances in centimeters.

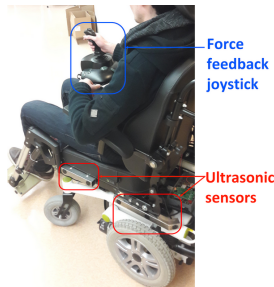

Fig. 8: Participant driving on the obstacle course. at the same location as in the simulation setup previously described (Fig. 5b).

The obstacle course we designed for this experiment (Fig. 7) is similar to the one used for the simulation (Fig. 4) but is more challenging as paths are narrower than the one used in simulation and it contains a turn around maneuver.

\section{B. Experiment protocol}

Participants were asked to drive a power wheelchair with the force feedback joystick (Fig. 8) on an obstacle course made with cardboard boxes (Fig. 7). Participants realized twice the course: once with haptic guidance and once without haptic guidance. The given instructions were

- driving along the obstacle course and go back,

- performing the obstacle course the faster they could without colliding with the obstacles.

For each trial, the haptic guidance module can be activated or not. We switched the order of activation of the haptic guidance for each participant in order to avoid the learning effect.

\section{Data Collection}

1) Haptic guidance solution data: We use ROS to automatically record data during the trials. Here we recorded user input, the related risk-free input computed by the algorithm and force magnitude and direction. We also recorded the number of collisions as well as time completion.

2) User feedback: After completing the obstacle courses, participants were asked to answer the USE questionnaire [23] which consists in a 30 items Likert scale. The Questionnaire is composed of 4 sections: Usefulness (U), Ease of Use (EU), Ease of Learning (EL) and Satisfaction (S).

\section{EXPERIMENT RESULTS}

This section presents the results of the experiment we performed. 4 able-bodied participants aged from 22 to 38 years old have volunteered to participate to this pilot study.

\section{A. User performance}

In order to evaluate the impact of the proposed haptic guidance solution on user performances while driving the power wheelchair, we recorded the number of collisions which occurred during the experiment with and without force feedback (Fig. 9a) as well as completion time.

As we can see, much fewer collisions occurred while haptic guidance was activated. With the haptic guidance activated, all participants collided the same obstacle. In fact, these collisions only occurred during a turn around maneuver which was really difficult as the space was very narrow compared to the length of the wheelchair. Moreover, there was no significant difference between time completion values with and without force feedback.

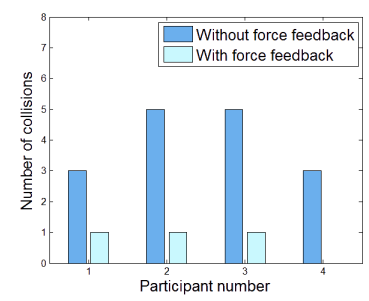

(a) Number of collisions.

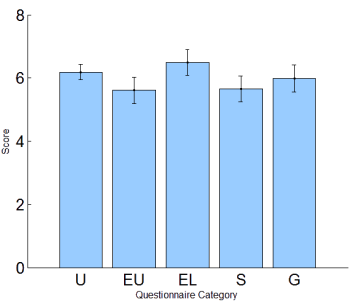

(b) USE Questionnaire results. Mean and standard deviation for each category of the USE Questionnaire.
Fig. 9: Experiment results.

\section{B. User feedback}

USE Questionnaire results are presented in Fig. 9b. Mean and standard deviation $(\mathrm{M}+/-\mathrm{SD})$ values are given for each category. Best score is for the ease of learning with $6.5+/$ 0.41 and worst score is for the ease of use with $5.61+/-$ 0.42 . Global questionnaire score is $5.99+/-0.43$.

Participants reported to be rather satisfied with the proposed haptic guidance solution. They felt the information given by the joystick was relevant and helpful for the navigation task. In particular, they reported having benefited from the haptic guidance while performing the turn around maneuver as it warned them while getting too close to obstacles and allowed them to better judge distances when obstacles were not easily visible as the force increased while approaching the obstacle with an unsafe joystick input. However, even if driving performances have been significantly increased, additional information provided by the force feedback joystick was not totally sufficient to avoid collisions during the turn around maneuver. Nevertheless, it globally incited participants to adapt their command and generally decreased the collision impact speed. Finally, participants did not report having felt any pain or discomfort while using the force feedback joystick but they reported to be unsatisfied with the haptic interface form factor itself: they found it difficult to manipulate because of its dimensions.

\section{DISCUSSION}

High scores obtained with the USE questionnaire show that the participants were rather satisfied with the haptic guidance solution. Moreover, they found it helpful, particularly for the turn around maneuver. As we expected, they felt that the joystick was giving them additional information about the environment. While approaching an obstacle, they perceived the force as an indication of the risk of collision inducing them to reduce their speed and to modify joystick direction. While maneuvering the wheelchair, they perceived 
the feedback as an inducement to move the joystick in the right direction.

These preliminary results show the validation of our haptic guidance solution as a tool for learning or rehabilitation purpose as it incited the participants to adapt speed and direction to avoid collisions and allowed them to better understand the environment around the wheelchair.

\section{CONCLUSION}

In this paper, we presented a haptic guidance solution for power wheelchair assisted navigation. In order to validate our solution, we asked 4 able-bodied participants to perform an experiment consisting in driving a power wheelchair equipped with a force feedback controller on an obstacle course with and without haptic guidance activated. This pilot study is mandatory to obtain an approval from an ethical committee in order to perform trials with people with disability. While driving the wheelchair on the obstacle course, the number of collisions significantly decreased while haptic guidance was activated, thus validating the proposed framework. This pilot study allowed us to identify the remaining shortcomings of our system. Evidently, the game controller we used to validate our robotic solution is not not adapted to wheelchair control. Hence, we started working on the design of a dedicated force feedback joystick adapted to user needs and which dimensions are compliant with wheelchair standards. In addition, in future work we would like to assess and compare our 3 navigation assistance solutions (obstacle avoidance only, haptic guidance only and haptic feedback) with more healthy participants before running clinical trials with power wheelchair regular users once approved by an ethical committee.

\section{ACKNOWLEDGEMENT}

The authors would like to thank Éric Bazin, Valentin Bureau and Nicolas Le Borgne from INSA Rennes who were of great help during the development process and experiments. Finally the authors would like to thank the people who participated to this study.

This work has been funded by Inria associated team ISI4NAVE and by Institut des Neurosciences Cliniques de Rennes (INCR). This work is carried out as part of the INTERREG VA FMA ADAPT project" Assistive Devices for empowering disAbled People through robotic Technologies http://adapt-project.com/index.php.". The Interreg FCE Programme is a European Territorial Cooperation programme that aims to fund high quality cooperation projects in the Channel border region between France and England. The Programme is funded by the European Regional Development Fund (ERDF).

\section{REFERENCES}

[1] A. Davies, L. D. Souza, and A. Frank, "Changes in the quality of life in severely disabled people following provision of powered indoor/outdoor chairs," Disability and Rehabilitation, vol. 25, no. 6, pp. 286-290, 2003.

[2] S. Evans, A. O. Frank, C. Neophytou, and L. De Souza, "Older adults' use of, and satisfaction with, electric powered indoor/outdoor wheelchairs," Age and ageing, vol. 36, no. 4, pp. 431-435, 2007.
[3] A. Rhoda, "Limitations in activity and participation experienced by stroke patients: A qualitative inquiry," South African Journal of Physiotherapy, vol. 68, no. 3, pp. 20-24, 2012.

[4] J. Cawood, S. Visagie, and G. Mji, "Impact of post-stroke impairments on activities and participation as experienced by stroke survivors in a western cape setting," South African Journal of Occupational Therapy, vol. 46, no. 2, pp. 10-15, 2016.

[5] Z. Li, S. Zhao, J. Duan, C. Y. Su, C. Yang, and X. Zhao, "Human cooperative wheelchair with brainmachine interaction based on shared control strategy," IEEE/ASME Transactions on Mechatronics, vol. 22, no. 1, pp. 185-195, Feb 2017.

[6] T. Panzarella, D. Schwesinger, and J. Spletzer, "Copilot: Autonomous doorway detection and traversal for electric powered wheelchairs," in Field and Service Robotics. Springer, 2016, pp. 233-248.

[7] M. F. Levin, H. Sveistrup, and S. Subramanian, "Feedback and virtual environments for motor learning and rehabilitation," Schedae, vol. 1, pp. 19-36, 2010.

[8] R. J. Stone, "Haptic feedback: A brief history from telepresence to virtual reality," in Haptic Human-Computer Interaction. Springer, 2001, pp. 1-16.

[9] S. Lichiardopol, "A survey on teleoperation," University of Eindhoven, Department Mechanical Engineering Dynamics and Control Group Eindhoven, 2007.

[10] J. K. Koehn and K. J. Kuchenbecker, "Surgeons and non-surgeons prefer haptic feedback of instrument vibrations during robotic surgery," Surgical endoscopy, vol. 29, no. 10, pp. 2970-2983, 2015.

[11] O. Lahav and D. Mioduser, "Haptic-feedback support for cognitive mapping of unknown spaces by people who are blind," International Journal of Human-Computer Studies, vol. 66, no. 1, pp. 23-35, 2008.

[12] M. G. Jones, G. Childers, B. Emig, J. Chevrier, H. Tan, V. Stevens, and J. List, "The efficacy of haptic simulations to teach students with visual impairments about temperature and pressure," Journal of Visual Impairment \& Blindness (Online), vol. 108, no. 1, p. 55, 2014.

[13] J. Broeren, M. Georgsson, M. Rydmark, and K. S. Sunnerhagen, "Virtual reality in stroke rehabilitation with the assistance of haptics and telemedicine," in Proceedings of the 4th International Conference on Disability, Virtual Reality and Associated Technologies, 2002, pp. 71-76.

[14] K.-S. Choi, C.-M. Chow, and K.-H. Lo, "A rehabilitation method with visual and haptic guidance for children with upper extremity disability," in International Conference on Computers for Handicapped Persons. Springer, 2010, pp. 77-84.

[15] L. M. Crespo and D. J. Reinkensmeyer, "Haptic guidance can enhance motor learning of a steering task," Journal of motor behavior, vol. 40, no. 6, pp. 545-557, 2008.

[16] L. Marchal-Crespo, J. Furumasu, and D. J. Reinkensmeyer, "A robotic wheelchair trainer: design overview and a feasibility study," Journal of neuroengineering and rehabilitation, vol. 7, no. 1, p. 40, 2010.

[17] X. Chen and S. K. Agrawal, "Assisting versus repelling force-feedback for human learning of a line following task," in Biomedical Robotics and Biomechatronics (BioRob), 2012 4th IEEE RAS \& EMBS International Conference on. IEEE, 2012, pp. 344-349.

[18] J. L. Protho, E. F. LoPresti, and D. M. Brienza, "An evaluation of an obstacle avoidance force feedback joystick," in RESNA 2000 Annual Conference, 2000, pp. 27-30.

[19] E. B. Vander Poorten, E. Demeester, E. Reekmans, J. Philips, A. Hüntemann, and J. De Schutter, "Powered wheelchair navigation assistance through kinematically correct environmental haptic feedback," in Robotics and Automation (ICRA), 2012 IEEE International Conference on. IEEE, 2012, pp. 3706-3712.

[20] M. Babel, F. Pasteau, S. Guégan, P. Gallien, B. Nicolas, B. Fraudet, S. Achille-Fauveau, and D. Guillard, "HandiViz project: clinical validation of a driving assistance for electrical wheelchair,' in IEEE Workshop On Advanced Robotics And Its Social Impacts (ARSO), Lyon, France, Jul. 2015.

[21] L. Devigne, M. Babel, F. Nouviale, V. Narayanan, F. Pasteau, and P. Gallien, "Design of an immersive simulator for assisted power wheelchair driving," in IEEE Int. Conf. on Rehabilitation Robotics, ICORR'17, 2017.

[22] L. Devigne, V. K. Narayanan, F. Pasteau, and M. Babel, "Low complex sensor-based shared control for power wheelchair navigation," in Intelligent Robots and Systems (IROS), 2016 IEEE/RSJ International Conference on. IEEE, 2016, pp. 5434-5439.

[23] A. M. Lund, "Measuring usability with the use questionnaire12," Usability interface, vol. 8, no. 2, pp. 3-6, 2001. 